\title{
EUROfusion
}

EUROFUSION WPJET1-PR(15)20

D. Tegnered et al.

\section{Comparative Gyrokinetic Analysis of JET Baseline H-mode Core Plasmas with Carbon Wall and ITER-Like Wall}

Preprint of Paper to be submitted for publication in

Plasma Physics and Controlled Fusion 
"This document is intended for publication in the open literature. It is made available on the clear understanding that it may not be further circulated and extracts or references may not be published prior to publication of the original when applicable, or without the consent of the Publications Officer, EUROfusion Programme Management Unit, Culham Science Centre, Abingdon, Oxon, OX14 3DB, UK or e-mail Publications.Officer@euro-fusion.org".

"Enquiries about Copyright and reproduction should be addressed to the Publications Officer, EUROfusion Programme Management Unit, Culham Science Centre, Abingdon, Oxon, OX14 3DB, UK or e-mail Publications.Officer@euro-fusion.org".

The contents of this preprint and all other EUROfusion Preprints, Reports and Conference Papers are available to view online free at http://www.euro-fusionscipub.org. This site has full search facilities and e-mail alert options. In the JET specific papers the diagrams contained within the PDFs on this site are hyperlinked. 


\title{
Comparative gyrokinetic analysis of JET baseline
} H-mode core plasmas with carbon wall and ITER-like wall

\author{
D. Tegnered ${ }^{1}$, P. Strand ${ }^{1}$, H. Nordman ${ }^{1}$, C. Giroud ${ }^{2}$, \\ Hyun-Tae Kim ${ }^{2}$, G.P. Maddison ${ }^{2}$, M. Romanelli ${ }^{2}$, G. Szepesi ${ }^{2}$ \\ and JET Contributors $\ddagger$ \\ EUROfusion Consortium, JET, Culham Science Centre, Abingdon, OX14 3DB, UK \\ ${ }^{1}$ Department of Earth and Space Sciences, Chalmers University of Technology, \\ SE-412 96 Göteborg, Sweden \\ ${ }^{2}$ CCFE, Culham Science Centre, Abingdon, OX14 3DB, UK \\ E-mail: tegnered@chalmers.se
}

12 2014, Saint Petersburg, Russia 


\section{Introduction}

Initial studies indicate that the interaction between the hot fusion plasma and the surrounding wall in magnetic fusion confinement devices can influence key plasma performance parameters like the energy confinement time. With the new ITER-like wall (ILW) at JET [1], the carbon wall (CW) has been replaced by a metal beryllium first wall and a tungsten divertor. To study the effect of the new ILW on confinement, a database has been created comprising a set of JET discharges with ILW and matched $\mathrm{C}$-wall shots using the same criteria as in [2]. The database contains both baseline H-mode and hybrid scenarios, at high and low delta. The ILW experimental program has already produced many interesting results which are not well understood $[2,3]$. In particular a deterioration in global confinement has been observed at JET in baseline H-mode experiments following the change from a from a CW to an ILW [4]. One cause of the deterioration is the high deuterium gas puffing rate necessary in ILW discharges in order to mitigate $\mathrm{W}$ accumulation. For low triangularity plasmas, this degradation of confinement with fuelling level was also previously observed for CW discharges [5]. The deterioration has been correlated by a degradation of pedestal confinement with lower electron temperatures at the top of the edge barrier region. This leads to lower electron temperature in the core, thereby changing the NBI heat deposition profiles in the core. As a result, the core energy confinement time has been influenced with lower electron energy confinement time and similar ion confinement time in the ILW case [4]. In the present work, gyrokinetic modelling of similar CW and ILW discharges is carried out in order to assess the differences seen in core confinement. The discharges have ion temperature data available and have been selected in order to match the average value of global controllable parameters within a reference time window during the flat top. Parameters are taken from interpretative TRANSP [6, 7] simulations. Transport due to Ion Temperature Gradient (ITG)/Trapped electron mode (TEM) $[8,9,10,11,12,13,14]$ turbulence is calculated using the gyrokinetic code GENE [15]. Both linear and nonlinear simulations are performed in a flux tube domain. The gyrokinetic simulations include finite $\beta$ effects, collisions, impurities, and rotational effects in realistic geometry. Linear sensitivity scans for the paired discharges are performed for plasma $\beta$, collisionality, safety factor, magnetic shear, impurity content and electron and ion temperature gradient. The differences in the energy flux and energy confinement times are investigated using nonlinear GENE simulations.

The remainder of the paper is organized as follows: In Sec. 2 the gyrokinetic model and the input parameters used are introduced. In Sec. 3 the linear sensitivity results are presented, followed by the nonlinear results in Sec. 4. Finally, in Sec. 5 follow the concluding remarks. 


\begin{tabular}{|c|c|c|c|c|c|}
\hline Shot number & $B(\mathrm{~T})$ & $T_{e}(\mathrm{keV})$ & $T_{i}(\mathrm{keV})$ & $n_{e}\left(10^{19} / \mathrm{m}^{3}\right)$ & $\Omega_{\text {tor }}\left(\mathrm{krads}^{-1}\right)$ \\
\hline \hline 74313 & 2.62 & 2.31 & 2.11 & 9.04 & 32 \\
\hline 85407 & 2.68 & 1.70 & 1.71 & 8.19 & 26 \\
\hline 74324 & 2.64 & 2.35 & 2.08 & 8.72 & 31 \\
\hline 85406 & 2.68 & 1.78 & 1.75 & 7.56 & 31 \\
\hline
\end{tabular}

Table 1: Discharge dimensional parameters of the four discharges.

\section{GENE simulations setup and discharge parameters}

GENE solves the nonlinear gyrokinetic Vlasov equations together with Maxwell's equations in order to find the distribution functions of the species, $f\left(\mathbf{R}, v_{\|}, \mu, t\right)$, the electrostatic potential, $\phi(\mathbf{x}, t)$ and the parallel components of the magnetic vector potential and magnetic field, $A_{\|}(\mathbf{x}, t)$ and $B_{\|}(\mathbf{x}, t)$. The coordinate system is aligned to the background magnetic field with $x$ as the radial coordinate, $y$ as the binormal coordinate, and $z$ as the parallel coordinate. Collisions are modelled using a linearised Landau-Boltzmann collision operator [16]. Magnetic fluctuations were included in all simulations. The pressure gradient, as used in the calculation of the curvature and $\nabla B$ drift, is set to be consistent with the density and temperature gradients and the plasma $\beta$. In this work, the Miller geometry model [17] is used. The Miller geometry model allows the magnetic geometry to be completely described by nine parameters. These parameters were extracted from numerical geometries reconstructed by the EFIT code [18]. For the linear simulations both an initial value solver and an eigenvalue solver that can find subdominant modes are used.

Two ITER-like wall discharges and two C-wall discharges with global parameters matched as closely as possible are analysed. The matched global parameters are the plasma current, the toroidal magnetic field, applied NBI power, average electron density, safety factor, and triangularity. The discharges are baseline H-mode with ion temperature and rotation measurements available through charge exchange spectroscopy. Discharge parameters are taken from TRANSP runs [7, 6] performed with electron density and temperature profiles from high resolution Thomson scattering measurements. One impurity species is included in the simulation, carbon for the carbon wall discharges and beryllium for the ITER-like wall discharges. The impurity density was calculated from $Z_{e f f}$, which is assumed to be constant over the whole radius [4]. The four discharges are analysed at $\rho=0.5$ where $\rho$ is the normalized toroidal flux coordinate. The baseline H-mode discharges are pair wise 74313 (CW), 85407 (ILW), 74324 (CW) and 85406 (ILW). In Figure 1 the time evolution of the discharges is shown. The relevant discharge parameters are shown in Table 1 (dimensional) and 2 (dimensionless). Radial temperature, density and rotational speed profiles are shown in Figure 2. The data is averaged over a one second time window and further smoothed in the radial direction. 

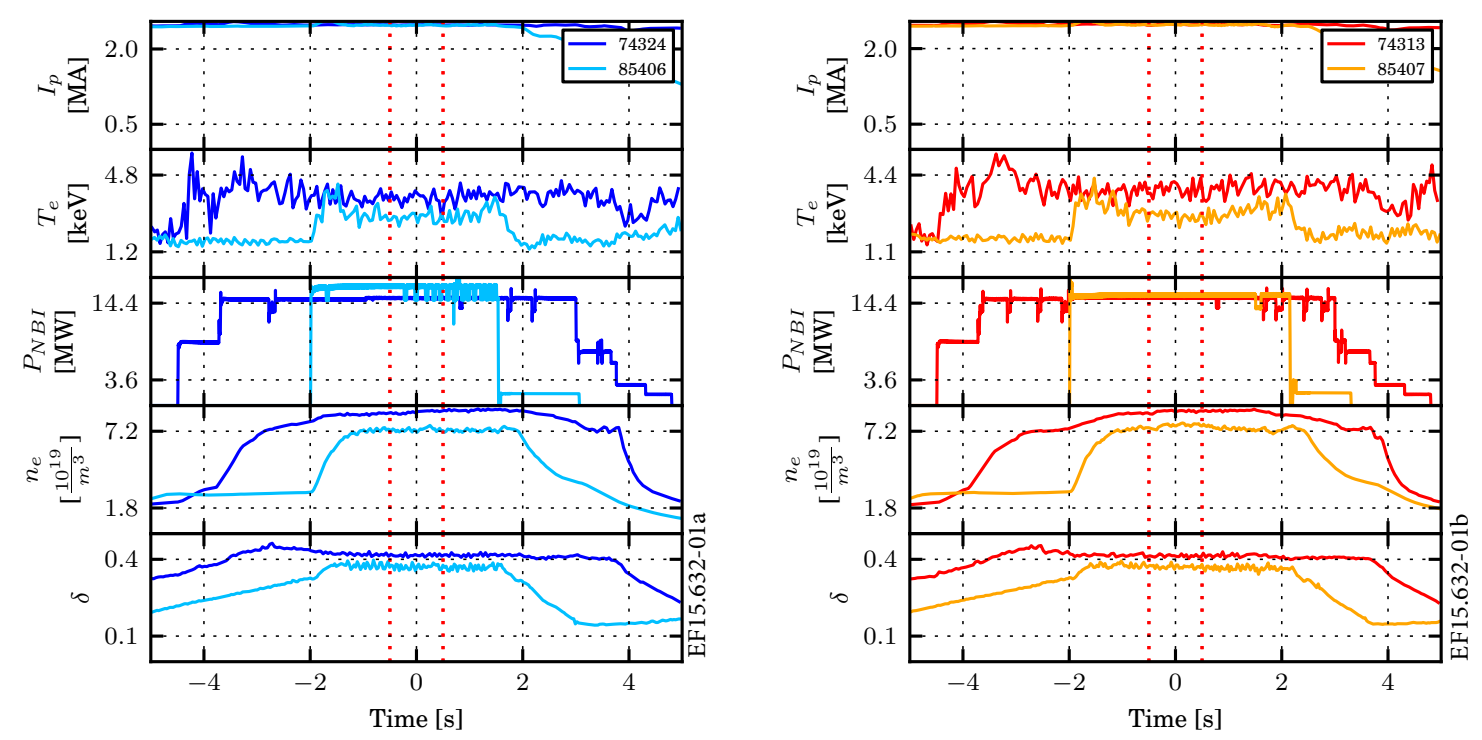

(a) CW discharge 74324 and ILW discharge 85407(b) CW discharge 74313 and ILW discharge 85406

Figure 1: Time evolution of the two pairs of matched discharges. Time point of analysis indicated with 0 .

\begin{tabular}{|c|c|c|c|c|c|c|c|c|c|c|}
\hline $\begin{array}{c}\text { Shot } \\
\text { number }\end{array}$ & $\hat{s}$ & $q$ & $T_{i} / T_{e}$ & $R / L_{T_{i}}$ & $R / L_{T_{e}}$ & $\beta(\%)$ & $\nu_{c}\left(10^{-3}\right)$ & $Z_{\text {eff }}$ & $\gamma_{\mathbf{E} \times \mathbf{B}}$ & $\delta$ \\
\hline \hline 74313 & 0.56 & 1.42 & 0.92 & 6.56 & 6.19 & 1.2 & 1.8 & 1.58 & 0.056 & 0.097 \\
\hline 85407 & 0.66 & 1.32 & 1.00 & 5.96 & 8.28 & 0.78 & 3.0 & 1.05 & 0.10 & 0.081 \\
\hline 74324 & 0.55 & 1.44 & 0.89 & 4.92 & 5.96 & 1.19 & 1.7 & 1.56 & 0.040 & 0.097 \\
\hline 85406 & 0.64 & 1.34 & 0.98 & 6.78 & 8.38 & 0.75 & 2.5 & 1.05 & 0.22 & 0.083 \\
\hline
\end{tabular}

Table 2: Discharge dimensionless parameters at $\rho=0.5$. Collision frequency calculated as $\nu_{c}=\pi \ln \Lambda e^{4} n_{e} R /\left(2^{3 / 2} T_{e}^{2}\right)$.

\section{Linear results}

The computational parameters used in the linear simulations are a resolution of $32 \times 24$ in the parallel and normal direction with 64 grid points in the parallel velocity direction and 16 magnetic moments. An initial value solver is typically used, in the cases where sub dominant modes are presented an eigenvalue solver is used. The linear ITG/TE mode stability of the two matched pairs is investigated at mid radius. Due to the experimental uncertainty in the value of $R / L_{T_{i}}$, the linear results are displayed in a scan over $R / L_{T_{i}}$. Figure $3 \mathrm{a}$ shows the growth rates and Fig. $3 \mathrm{~b}$ the corresponding eigenfrequencies at $k_{y} \rho_{s}=0.3$. As observed, the turbulence is ITG dominated for $R / L_{T_{i}}>4\left(\omega_{r}>0\right)$ for the ILW discharges and TEM dominated for lower $R / L_{T_{i}}$ while for the CW discharges 


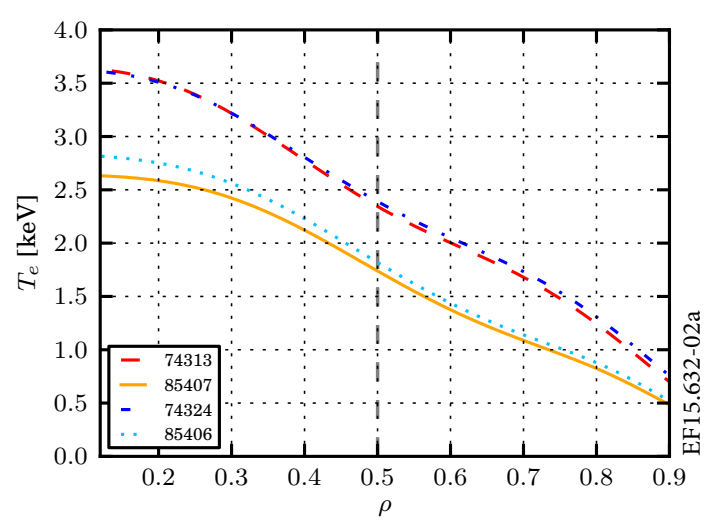

(a) Smoothed electron temperature profiles

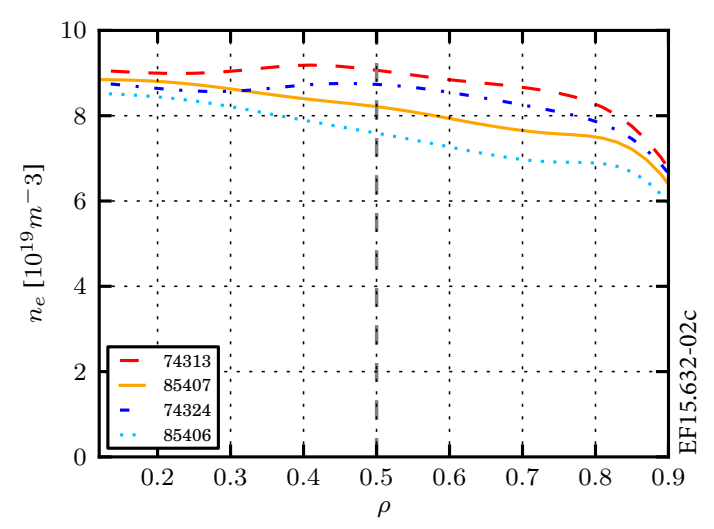

(c) Smoothed electron density profiles

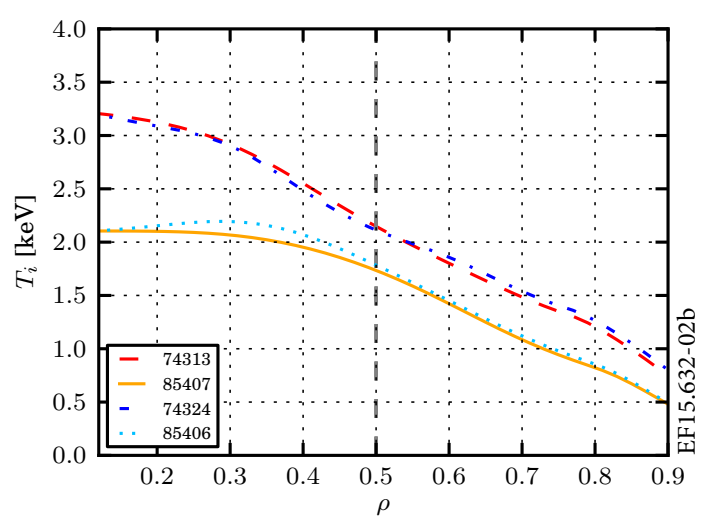

(b) Smoothed ion temperature profiles

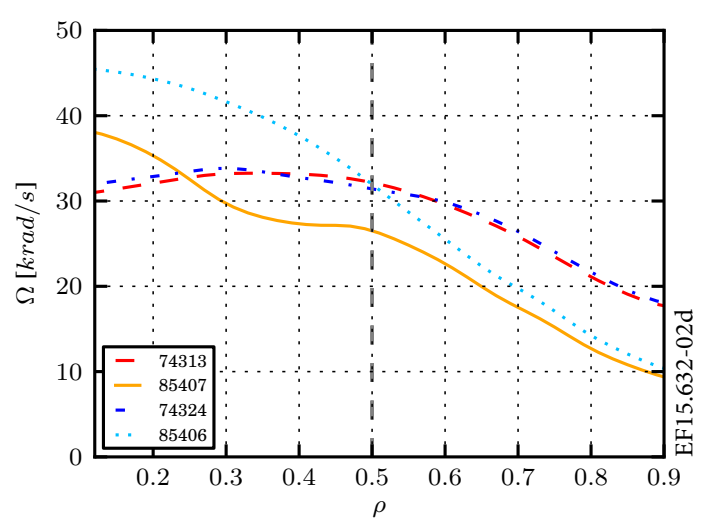

(d) Smoothed rotation profiles

Figure 2: Density and temperature profiles from time averaged and smoothed TRANSP data. Values are averaged between $11.5 \mathrm{~s}$ and $12.5 \mathrm{~s}$ for the $\mathrm{C}$-wall discharges and $19.5 \mathrm{~s}$ and $20.5 \mathrm{~s}$ for the ITER-like wall discharges.

the TE mode is not excited. We have verified that the results are similar for other values of $k_{y} \rho_{s}$ around the maximum growth rate which occurs at around $k_{y} \rho_{s}=0.3$. The ITG threshold is slightly lower for the ILW discharges and the normalized growth rates are smaller at the same $R / L_{T_{i}}$. For the experimental values of $R / L_{T_{i}}$ (marked in Fig. 3), we obtain $\gamma_{I T G}=0.16$ for ILW discharge 85407 and $\gamma_{I T G}=0.11$ for the matched CW discharge in units of $c_{s} / R$. Similar results are obtained for the other pair of discharges.

In order to investigate the physics behind the difference in linear stability for the matched pairs, a sensitivity study is performed with respect to the key dimensionless parameters. The analysis include variations in plasma $\beta$, collisionality, magnetic shear, Shafranov shift, $R / L_{T_{e}}$, ion to electron temperature ratio, safety factor, impurity content and triangularity. The parameters are varied around the experimental values with up to $20 \%$. The analysis is limited to one of the discharge pairs, but we have confirmed that the conclusions are similar for the pairs under investigation. 


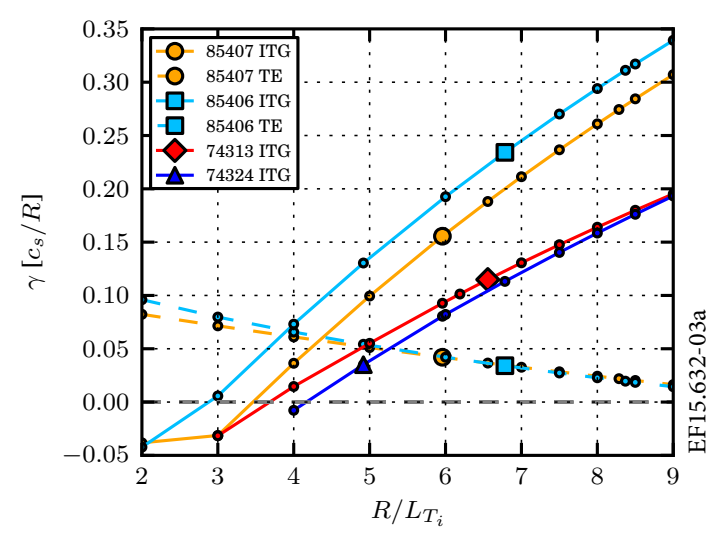

(a) Linear growth rate with $k_{y} \rho_{s}=0.3$

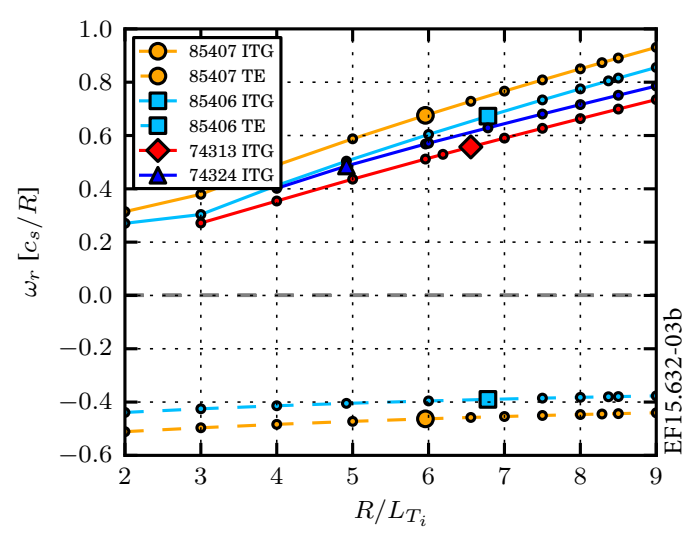

(b) Linear eigenfrequency with $k_{y} \rho_{s}=0.3$

Figure 3: Linear $R / L_{T_{i}}$ scans for the four discharges at $k_{y} \rho_{s}=0.3$. Experimental $R / L_{T_{i}}$ indicated.

First, in Figure 4, the growth rate spectrum is shown with plasma $\beta$ as a parameter. The results show the well known linear stabilization of the ITG mode with plasma $\beta$. The experimental values are $\beta=0.78 \%$ for the ILW discharge and $\beta=1.2 \%$ for the $\mathrm{C}$-wall case. The reason for the larger $\beta$ value in the $\mathrm{C}$-wall discharge can be traced to the difference in pedestal hight which is significantly lower in the ILW discharges.

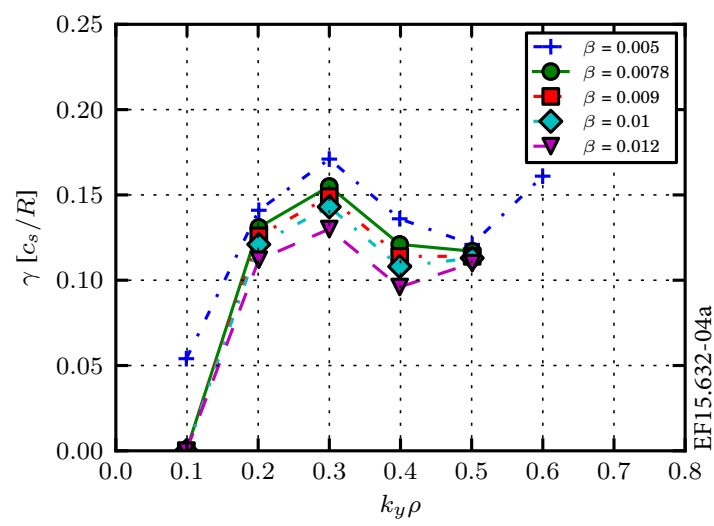

(a) ILW discharge 85407

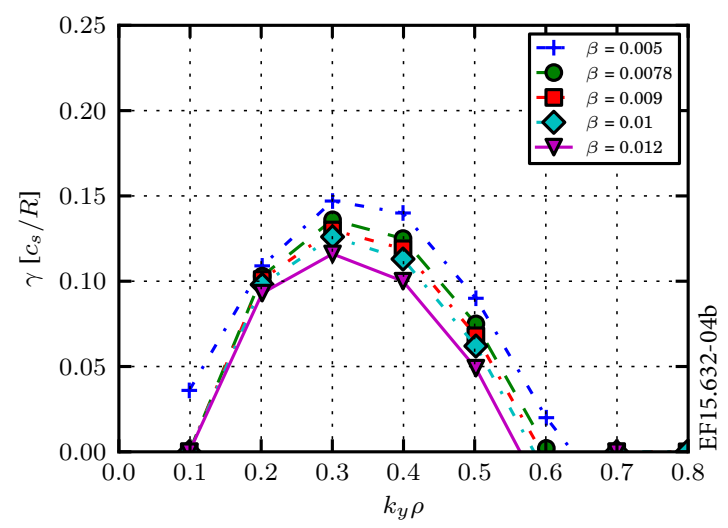

(b) CW discharge 74313

Figure 4: Scaling of eigenvalue spectra with $\beta$

The difference in plasma $\beta$ between the matched discharges also has an effect on the magnetic geometry through the Shafranov shift. Hence, the Shafranov shift is larger for the C-wall case which enhances the stability of the ITG modes, as is shown in Figure 5 .

Next, the sensitivity with respect to magnetic shear is displayed. Magnetic shear is slightly destabilizing for ITG modes in the parameter regimes considered. As can 


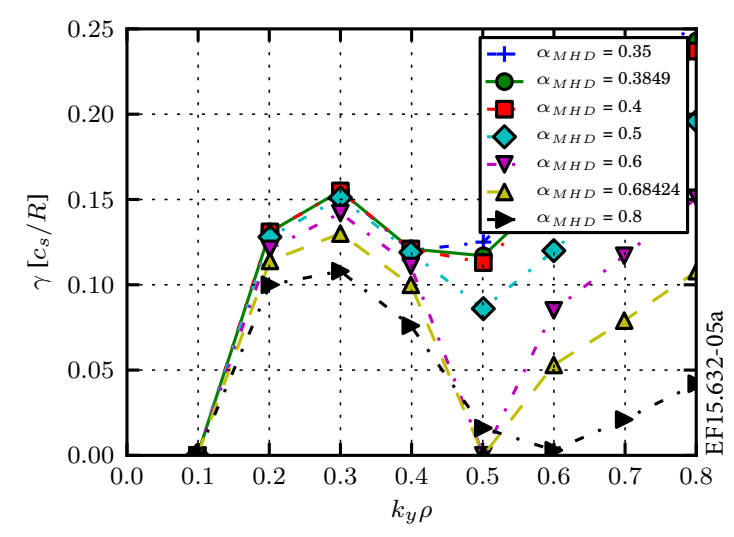

(a) ILW discharge 85407

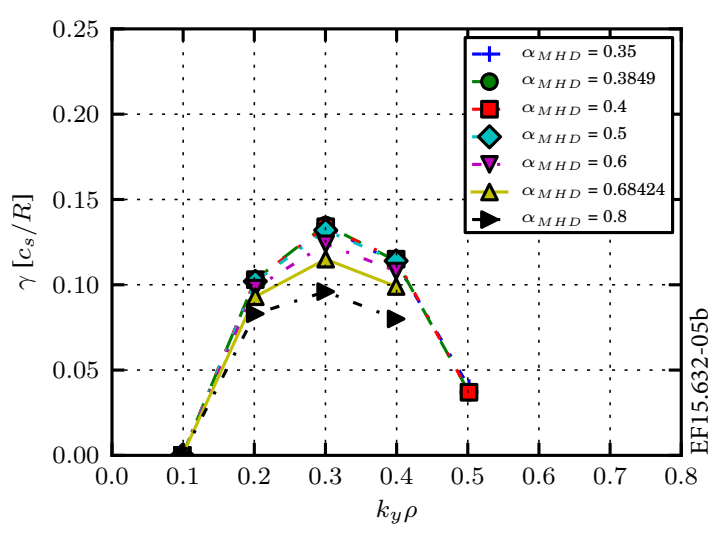

(b) CW discharge 74313

Figure 5: Scaling of eigenvalue spectra with $\alpha_{M H D}$

be seen in Figure 6, the magnetic shear is larger for the ILW discharge, with $\hat{s}=0.66$ whereas $\hat{s}=0.56$ for the C-wall case.

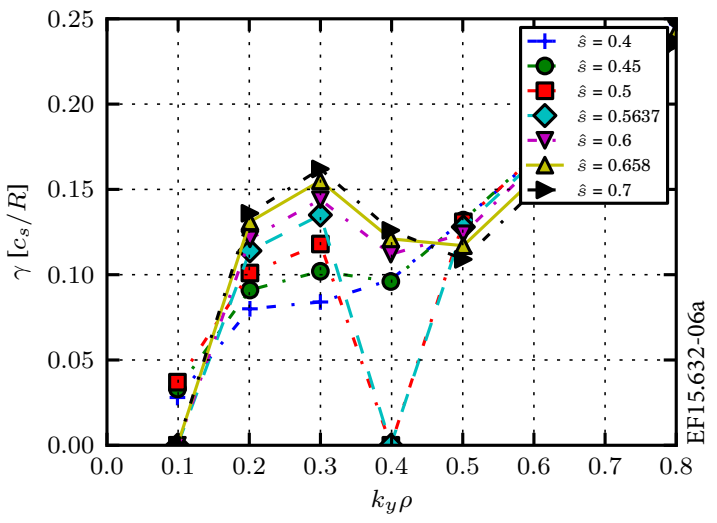

(a) ILW discharge 85407

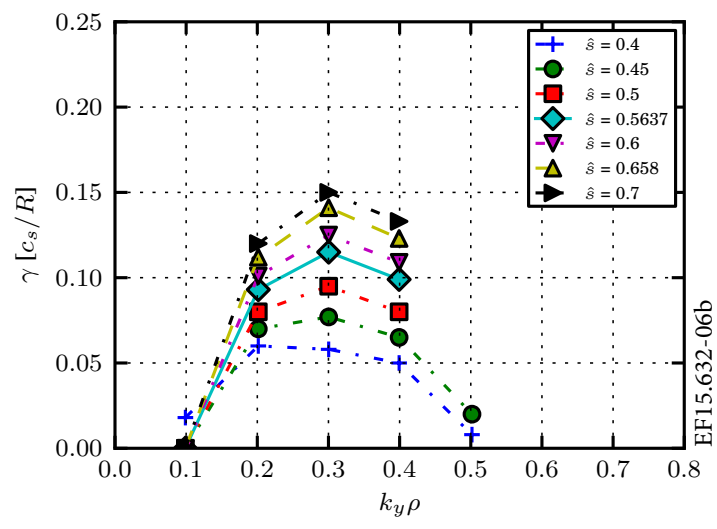

(b) CW discharge 74313

Figure 6: Scaling of eigenvalue spectra with $\hat{s}$

In Figure 7 the destabilizing effect of the electron temperature gradient on the ITG stability is illustrated. The electron temperature gradient is larger for the ILW discharges $\left(R / L_{T_{e}}=8.3\right.$ versus $R / L_{T_{e}}=6.2$ in the C-wall case) which destabilizes both the ITG mode and the TE mode.

Figure 8 displays the corresponding growth rate spectra with collisionality given in Gaussian units with $\nu_{c}=\pi \ln \Lambda e^{4} n_{e} R /\left(2^{3 / 2} T_{e}^{2}\right)$ as a parameter. The collisionality is stabilizing for both discharges, with $\nu_{c}=0.003$ for the ILW case and $\nu_{c}=0.0019$ for CW. Since the collisionality is larger for the ILW discharges the relative effect of collisionality is stabilizing for ILW discharges. The reason for the larger collisionality in the ILW case is the lower temperatures in the ILW discharge. 


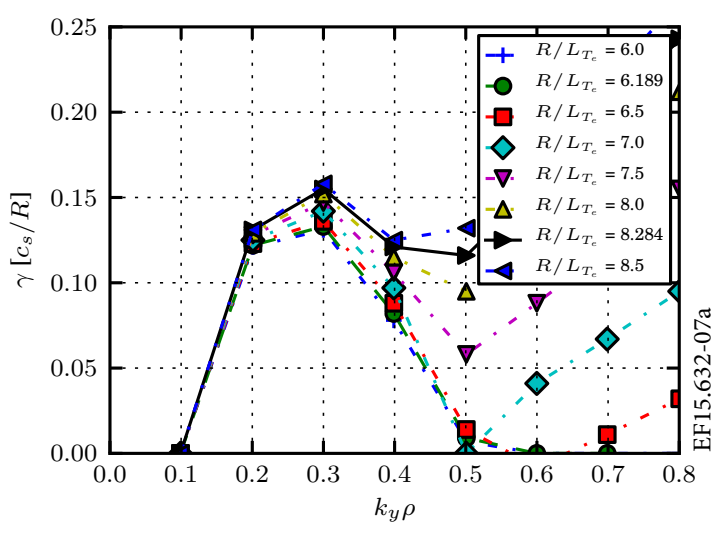

(a) ILW discharge 85407

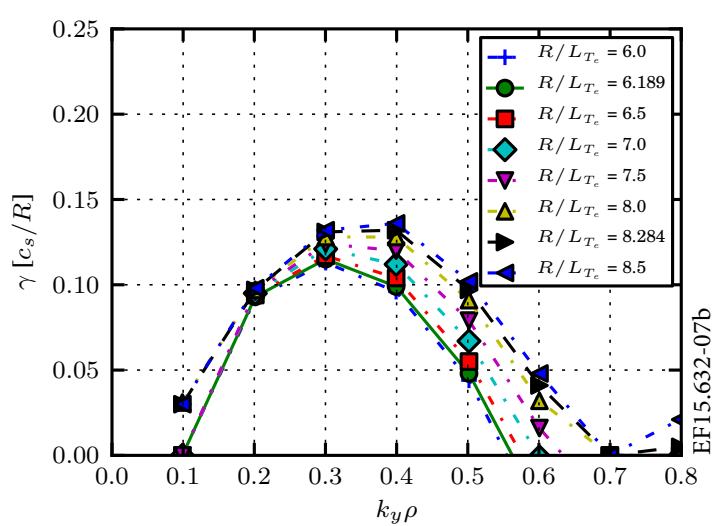

(b) CW discharge 74313

Figure 7: Scaling of eigenvalue spectra with $R / L_{T_{e}}$

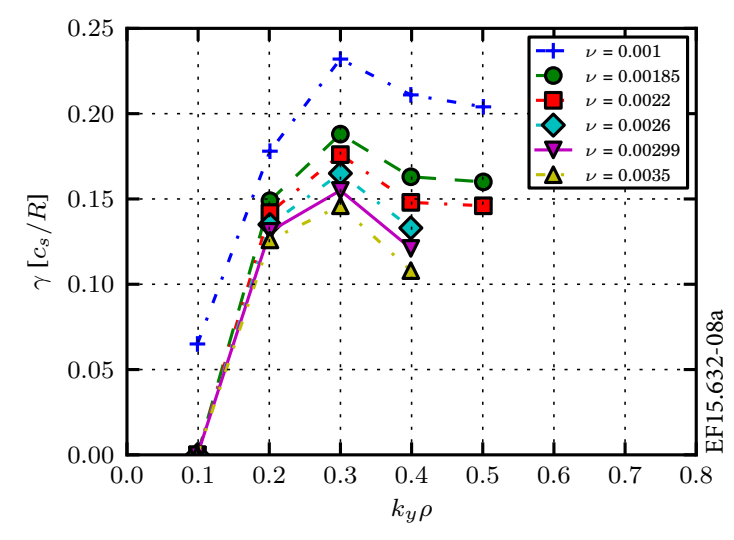

(a) ILW discharge 85407

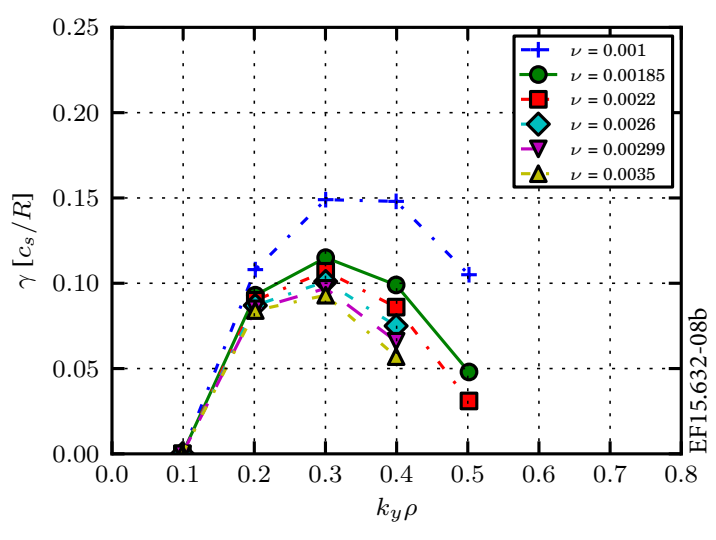

(b) CW discharge 74313

Figure 8: Scaling of eigenvalue spectra with $\nu_{c}$

Finally, the effect of temperature ratio and impurity content on linear stability is investigated. The ion to electron temperature ratio is slightly larger for the ILW discharges $\left(T_{i} / T_{e}=1.0\right.$ versus $T_{i} / T_{e}=0.91$ in the CW case). This is stabilizing the ITG mode but destabilizing the TE mode, as shown in Figure 9.

The impurity fraction and composition $(\mathrm{C}$ versus $\mathrm{Be})$ is different in the matched pairs. It is well established that the impurity fraction is lower in the ILW discharges [3]. The impurities have a stabilizing influence on the ITG mode, mainly through main ion dilution. The result is a slightly more stable ITG mode in the C-wall case.

In summary, the ILW versus C-wall pairs considered are not perfectly matched with respect to dimensionless parameters. This leads to differences in linear stability of the main instabilities in the discharges. The reason for the mismatch in many parameters is related to the difference in pedestal height. This difference in the edge region translates 


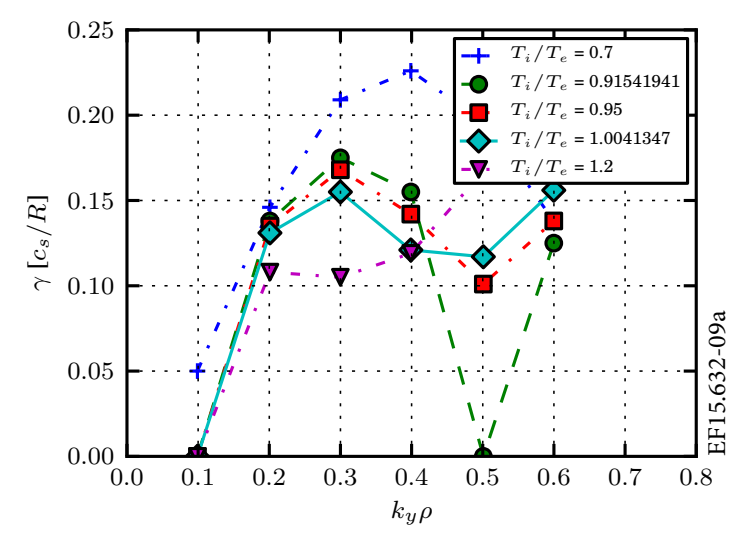

(a) ILW discharge 85407

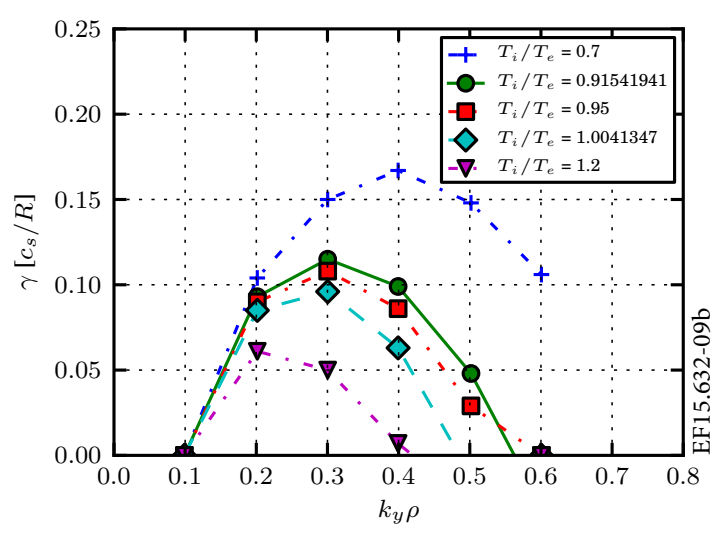

(b) CW discharge 74313

Figure 9: Scaling of eigenvalue spectra with $T_{i} / T_{e}$

into differences in the core of key parameters like $\beta$, Shafranov shift, and collisionality. These differences are expected to disappear if the pedestal confinement is recovered, e.g. through $\mathrm{N}$ seeding [19]. The difference in impurity content between the pairs leads to a slightly more stable situation in the $\mathrm{C}$-wall case which should remain even if the pedestals are similar.

In Figure 10, the effect of the difference in dimensionless parameters on the linear stability is summarized. The figure shows the relative change in the ITG growth rate when the values of the parameters in one discharge is changed to that of the corresponding paired discharge. As seen, the mismatch in $\beta$, Shafranov shift, magnetic shear, and electron temperature gradient serve to destabilize the ILW discharges relative to the $\mathrm{CW}$ discharges while the mismatch in collisionality and ion to electron temperature ratio tend to stabilize the ILW discharges. The difference in the safety factor and triangularity did not substantially change the linear stability properties.

\section{Nonlinear results}

For the nonlinear GENE simulations, a simulation domain in the perpendicular plane of $\left[L_{x}, L_{y}\right]=[146,126]$ were used, with a resolution of $\left[n_{x}, n_{y}\right]=[96,48]$. In the parallel direction 32 grid points were used, and in the parallel velocity direction 64 grid points, and 16 magnetic moments. The simulations were typically run up to a simulation time of $t=300 \mathrm{R} / \mathrm{c}_{\mathrm{s}}$ where $R$ is the major radius and $c_{s}=\sqrt{T_{e} / m_{i}}$. The resolution and simulation domain are checked through convergence tests. The two matched pairs of ILW and CW discharges are simulated with input data taken at $\rho=0.5$. The simulations included effects of collisions, finite $\beta$, Miller equilibrium and impurity species, with an impurity concentration of $0.4 \%$ of Be in the ILW discharges and $1.9 \%$ of $\mathrm{C}$ in the $\mathrm{CW}$ discharges. In order to quantify the effects of rotation, its effect is included in one simulation of each discharge. For some of the simulations, a higher $R / L_{T_{i}}$ than the 


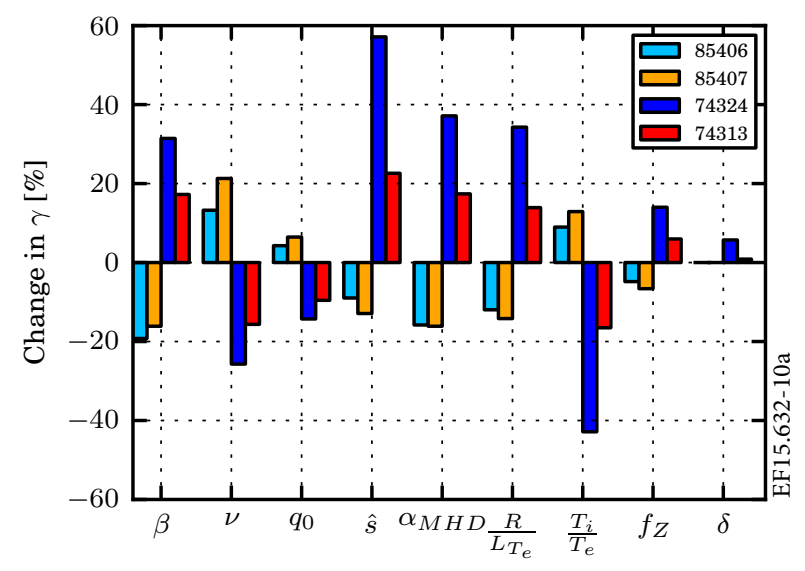

Figure 10: Growth rate change at $k_{y} \rho_{s}=0.3$

experimental was chosen because of the strong stabilizing effect of the ExB shear. As can be seen in Figure 14b, this results in a reduction in the ion heat flux of around $20 \%$. For these simulation, both the effect from the toroidal shear and Coriolis and centrifugal forces are included. Suprathermal pressure from fast ions, which has been reported to lead to a significant reduction in the ion heat flux in gyrokinetic simulations of JET discharges [20, 21, 22], is not included in the present simulations. While it was shown in [20] that effects of fast ions were important at low radia $(\rho=0.3)$ and low magnetic shear, a weak effect was observed at larger radia and magnetic shear relevant to the present case.

Due to the large uncertainty in the parameter $R / L_{T_{i}}$, the nonlinear simulations of the ILW and CW discharges are performed as scans over $R / L_{T_{i}}$. A typical result for the time series and flux spectra is shown in Figure 11 and 12 for the case with $R / L_{T_{i}}=R / L_{T_{e}}$, for the matched pairs 85407 (ILW), 74313 (CW) and 85406 (ILW), $74324(\mathrm{CW})$. In order to investigate any differences in flux spectra between the matched pairs, the mean $k_{y} \rho_{s}$ for the ion heat flux was calculated along with a measure of the width of the spectra. The width is taken as the wavenumbers responsible for $25 \%$ of the flux over and under the indicated mean. The result is shown in Figure 13. As seen, the differences in mean wavenumber and spectrum width between the ILW and CW discharges are small. Figure 14 shows the scaling of ion and electron energy flux with $R / L_{T_{i}}$ in both normalized gyroBohm units and SI units. The electron temperature gradient is here fixed at the experimental value. The error margin is obtained from the time series, taking the statistical inefficiency of the data into account. An estimate of the stiffness is obtained from the normalized fluxes in Figure 14. As observed, the stiffness of the ILW discharges is larger than the matched ILW-discharges. In non normalized units the heat flux for all the four discharges is comparable at the same $R / L_{T_{i}}$. The ion heat flux is larger than the electron heat flux as expected for ITG dominated discharges. In Figure 14b, the ion heat flux at $\rho=0.5$ taken from the 


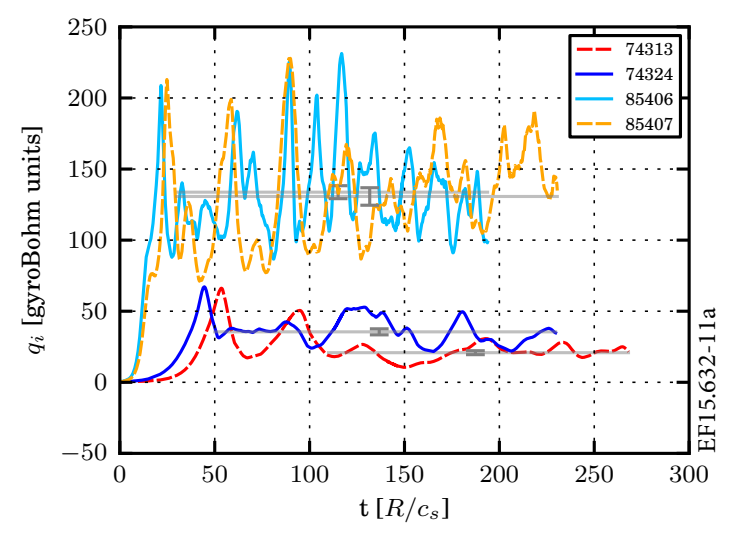

(a) Normalized ion heat flux

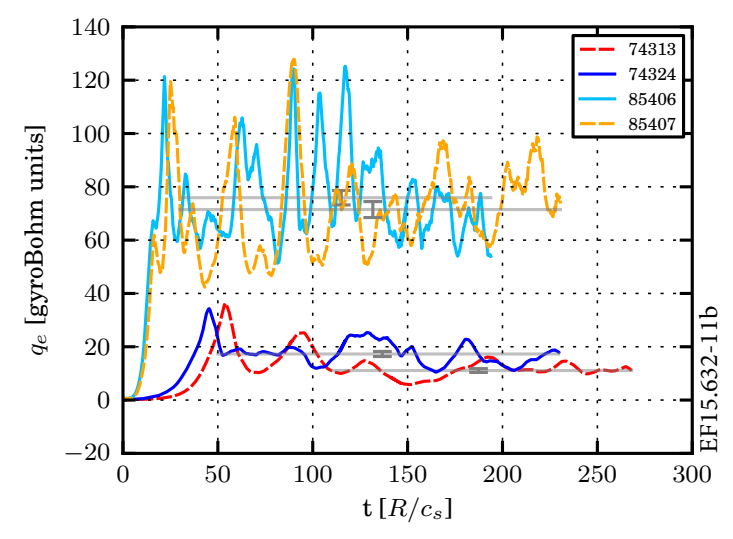

(b) Normalized electron heat flux

Figure 11: Time series data of the normalized ion and electron heat flux for the two pairs of CW and ILW discharges.

corresponding TRANSP runs is also shown. For the discharges at lower $R / L_{T_{i}}$ the experimental heat flux is comparable to the simulated flux while for the discharges at higher $R / L_{T_{i}}$, the simulated ion heat flux is a factor $\sim 3$ higher. The discrepancy between the experimental and simulated fluxes can be explained by the uncertainty in the input parameters, in particular the uncertainty in the ion temperature gradient is large for the ILW discharges. The results follow the linear trends in that the linearly more unstable ILW discharges show significantly larger normalized fluxes. This is quantified in Table 3 where the ion and electron heat fluxes and heat diffusivities are shown together with the linear ITG growth rates for the four discharges.

The core energy confinement times in the volume within $\rho=0.5$ are calculated per species as

$$
\tau_{\text {core }}^{j}(\rho<0.5)=\frac{\frac{3}{2} k_{B} \int_{0}^{V^{\prime}(\rho=0.5)} n_{j}(\rho) T_{j}(\rho) d V}{q_{j}(\rho=0.5)} .
$$

The results are shown in Figure 15. The electron energy confinement times are shorter for the ILW discharges while the ion energy confinement times are similar. As noted, the heat fluxes in SI units are similar at the same $R / L_{T_{i}}$, comparing the CW and ILW discharges. The shorter electron energy confinement times are thus due to the larger difference in $T_{e}$ than $T_{i}$ in the plasma within $\rho<0.5$ comparing the ILW and $\mathrm{CW}$ discharges, as seen in Figure $2 \mathrm{a}$ and $2 \mathrm{~b}$. These conclusions are in line with the experimental analysis of [4]; the difference can be attributed to the difference in NBI heating power deposited to the electrons and ions in the ILW versus CW cases. The fraction of total NBI power deposited to the electrons is larger for ILW discharges as compared to the CW discharges. This is a result of the lower edge $T_{e}$ in the ILW discharges. 


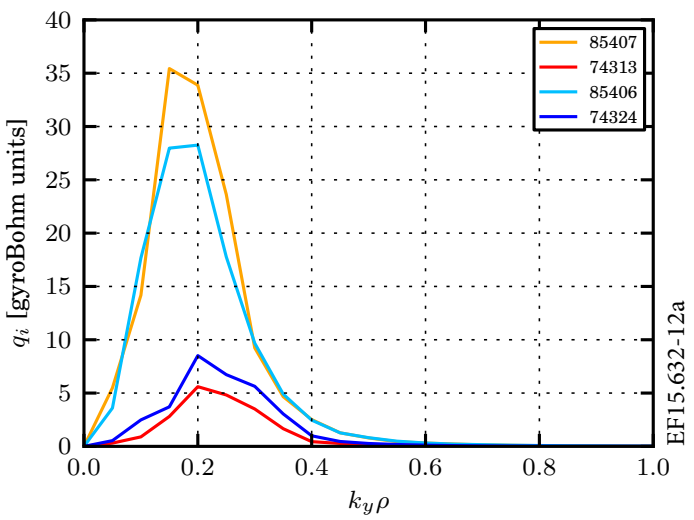

(a) Ion heat flux spectrum

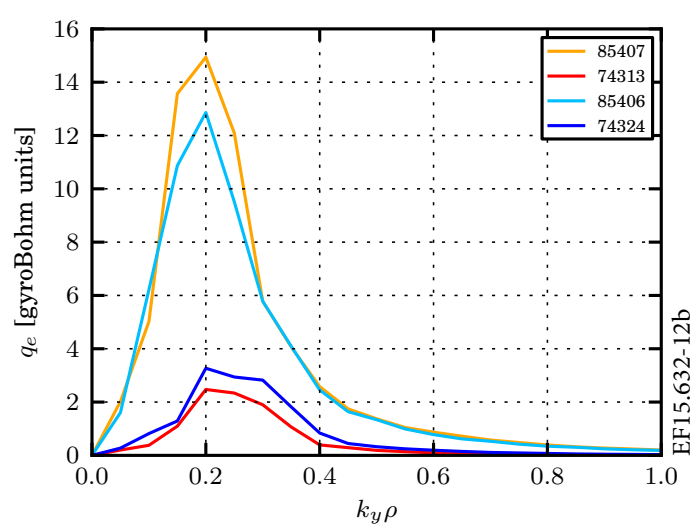

(b) Electron heat flux spectrum

Figure 12: Time averaged heat flux spectra

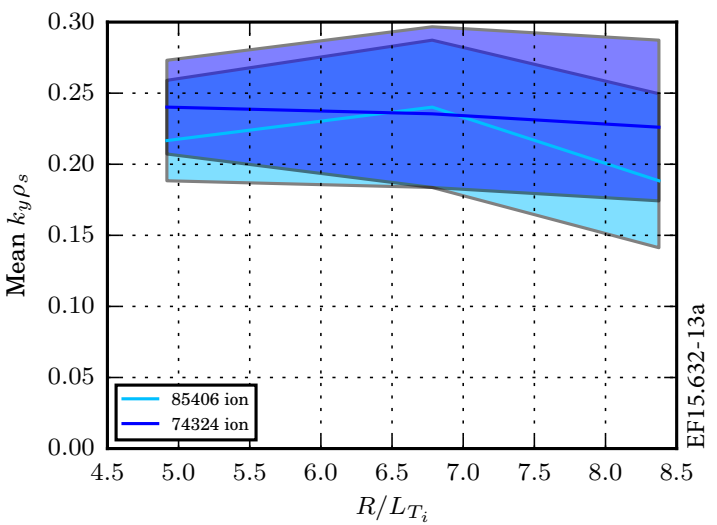

(a) 85406 and 74324

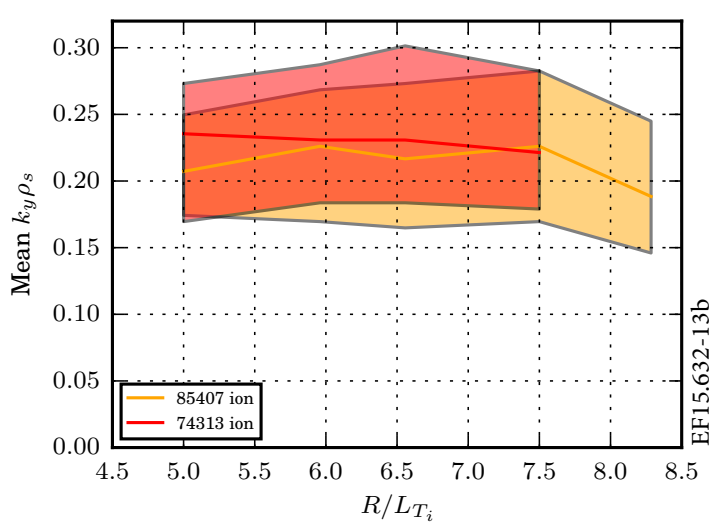

(b) 85407 and 74313

Figure 13: The mean wavenumber and width of the ion flux spectra for the two pairs of discharges, scaled with $R / L_{T_{i}}$.

\begin{tabular}{|c|c|c|c|c|c|c|c|}
\hline Shot number & $R / L_{T_{i}}$ & $R / L_{T_{e}}$ & $q_{i}$ & $q_{e}$ & $\chi_{i}$ & $\chi_{e}$ & $\gamma_{I T G}$ \\
\hline \hline 74313 & 6.56 & 6.19 & $27.7 \pm 1.7$ & $14.6 \pm 0.8$ & 5.1 & 2.3 & 0.11 \\
\hline 85407 & 5.96 & 8.28 & $39.9 \pm 2.2$ & $24.4 \pm 1.3$ & 6.8 & 2.9 & 0.16 \\
\hline 74324 & 4.92 & 5.96 & $10.8 \pm 1.8$ & $5.51 \pm 0.89$ & 3.0 & 1.0 & 0.035 \\
\hline 86406 & 6.78 & 8.37 & $71.6 \pm 4.9$ & $44.4 \pm 3.0$ & 9.9 & 4.8 & 0.23 \\
\hline
\end{tabular}

Table 3: Linear and nonlinear results for the four discharges with experimental ion and electron temperature gradients. The heat fluxes and heat diffusivities are given in gyroBohm units, $c_{s} n_{e} T_{e} \rho_{s}^{2} / R^{2}$ and $c_{s} \rho_{s}^{2} / R$, respectively. The linear data is for $k_{y} \rho_{s}=0.3$ in units of $c_{s} / R$. 


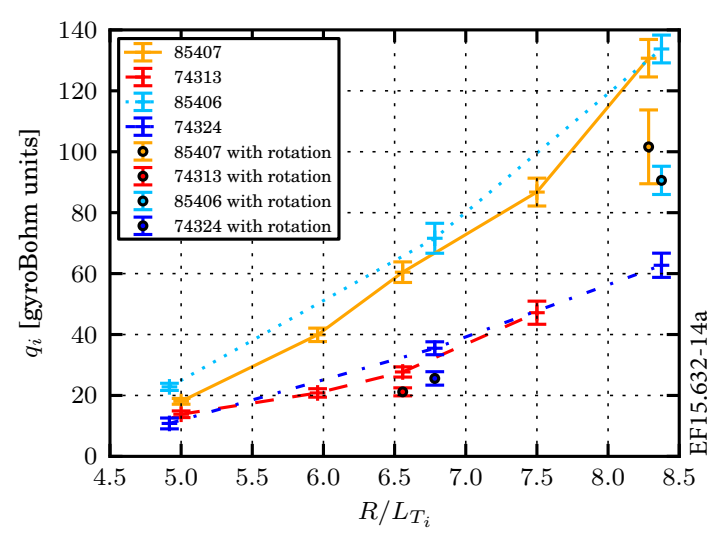

(a) Ion heat flux in normalized units

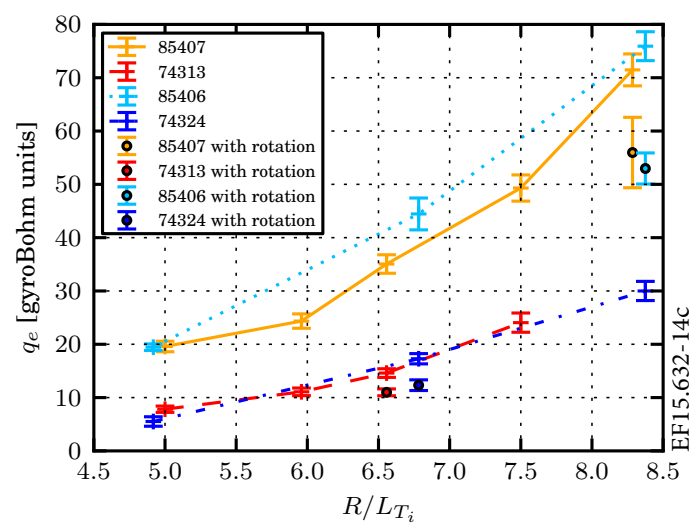

(c) Electron heat flux in normalized units

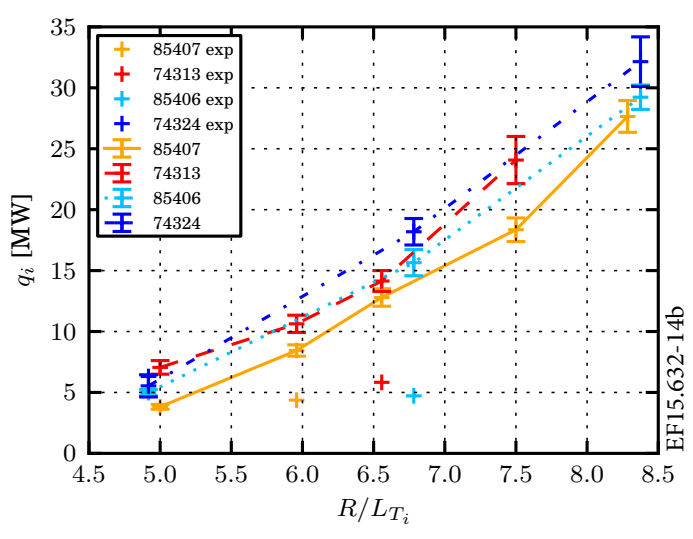

(b) Ion heat flux in MW

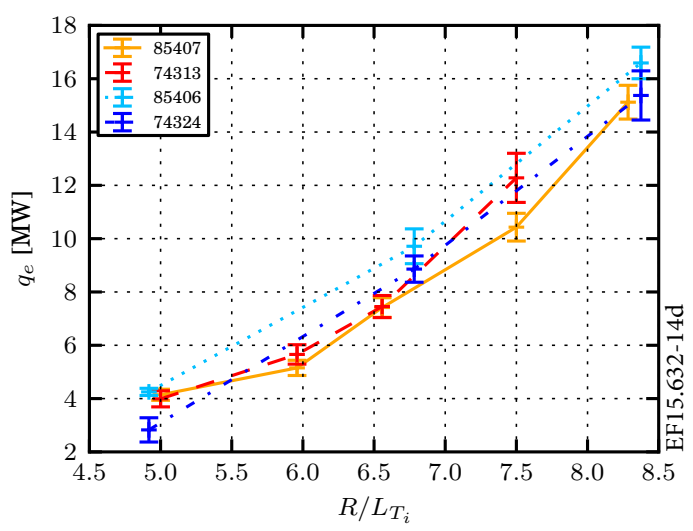

(d) Electron heat flux in MW

Figure 14: Nonlinear $R / L_{T_{i}}$ scans, electron and ion heat flux

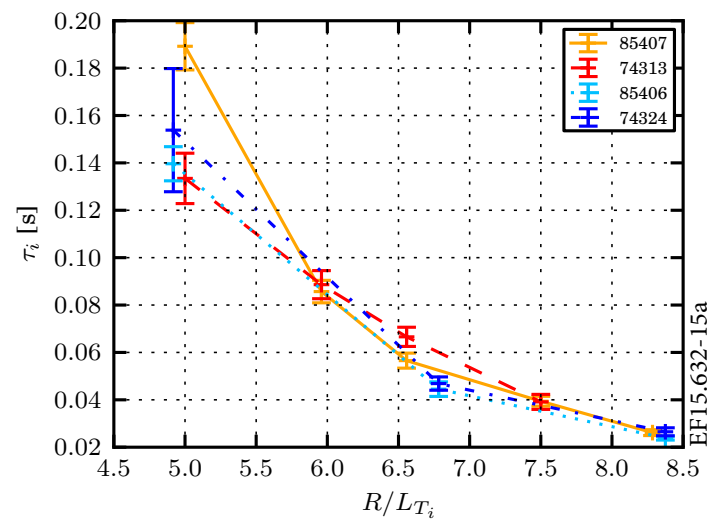

(a) Ion energy confinement times

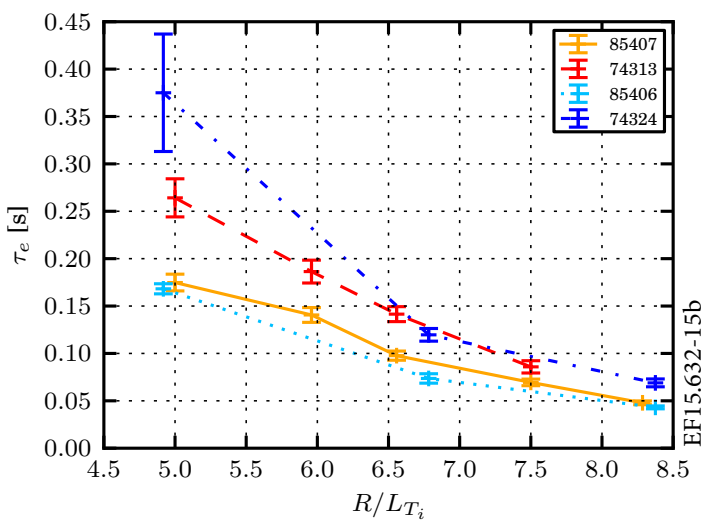

(b) Electron energy confinement times

Figure 15: Ion and electron energy confinement times in the volume within $\rho<0.5$ for the four discharges seen in $R / L_{T_{i}}$ scans. 


\section{Conclusion}

In the present paper, the linear stability and nonlinear fluxes of two pairs of matched ILW and CW baseline ITG dominated H-mode discharges were studied at mid radius using gyrokinetic simulations. The gyrokinetic simulations were performed using the GENE code in a flux tube domain. The simulations included effects of collisions, finite $\beta$ and impurities, Be for the ILW discharges and $\mathrm{C}$ for the $\mathrm{CW}$ discharges. The profile data was taken from TRANSP runs with electron and ion temperature measurements. A realistic Miller geometry description was used with parameters extracted from EFIT reconstructions. The focus was on explaining the differences seen in core confinement in baseline H-mode plasmas since the change of plasma facing components from a carbon wall to a metal wall. Experimentally, this has resulted in a degradation of the pedestal confinement with lower electron temperatures at the top of the edge barrier region. The linear sensitivity scans showed that the relative change in key plasma parameters between the ILW and CW discharges had a significant effect on the ITG/TE mode stability. The relative change in plasma $\beta$, Shafranov shift, $R / L_{T_{e}}$ and magnetic shear served to destabilize the ILW discharges, while the relative change in collisionality and ion-to-electron temperature ratio served to stabilize them. The total effect of these parameter mismatches was that the ILW discharges were destabilized compared to the CW discharges at all $k_{y} \rho_{s}$. The nonlinear results followed the linear ones in that the ILW discharges show higher normalized heat fluxes at both comparable and experimental $R / L_{T_{i}}$. The ion energy confinement times were similar, comparing the CW and ILW discharges while the electron energy confinement times were shorter for the ILW discharges which is in line with experimental analysis. These results indicate that the core confinement in the ILW discharges was affected by changes in key plasma parameters due to the degradation of the edge pedestal if compared to $\mathrm{CW}$ discharges. Hence, we expect the core confinement in the ILW discharges to be improved if the edge pedestals were recovered.

\section{Acknowledgements}

The simulations were performed on resources provided by the Swedish National Infrastructure for Computing (SNIC) at PDC Centre for High Performance Computing (PDC-HPC), on the HELIOS supercomputer system at Computational Simulation Centre of International Fusion Energy Research Centre (IFERC-CSC), Aomori, Japan, under the Broader Approach collaboration between Euratom and Japan, implemented by Fusion for Energy and JAEA, and on the supercomputer JUROPA at Jülich Supercomputing Centre (JSC). This work was funded by a grant from The Swedish Research Council (C0338001). This work has been carried out within the framework of the EUROfusion Consortium and has received funding from the Euratom research and training programme 2014-2018 under grant agreement No 633053. The views and opinions expressed herein do not necessarily reflect those of the European Commission. 


\section{References}

[1] GF Matthews, P Edwards, H Greuner, A Loving, H Maier, Ph Mertens, V Philipps, V Riccardo, M Rubel, C Ruset, et al. Physica Scripta, 2009(T138):014030, 2009.

[2] MNA Beurskens, J Schweinzer, C Angioni, A Burckhart, CD Challis, I Chapman, R Fischer, J Flanagan, L Frassinetti, C Giroud, et al. Plasma Physics and Controlled Fusion, 55(12):124043, 2013.

[3] MNA Beurskens, L Frassinetti, C Challis, C Giroud, S Saarelma, B Alper, C Angioni, P Bilkova, C Bourdelle, S Brezinsek, P Buratti, G Calabro, T Eich, J Flanagan, E Giovannozzi, M Groth, J Hobirk, E Joffrin, MJ Leyland, P Lomas, E de la Luna, M Kempenaars, G Maddison, C Maggi, P Mantica, M Maslov, G Matthews, ML Mayoral, R Neu, I Nunes, T Osborne, F Rimini, R Scannell, ER Solano, PB Snyder, I Voitsekhovitch, P de Vries, and JET-EFDA Contributors. Nuclear Fusion, 54(4):043001, 2014.

[4] Hyun-Tae Kim, M Romanelli, I Voitsekhovitch, T Koskela, J Conboy, C Giroud, G Maddison, E Joffrin, et al. Plasma Physics and Controlled Fusion, 57(6):065002, 2015.

[5] R Neu, G Arnoux, M Beurskens, V Bobkov, S Brezinsek, J Bucalossi, G Calabro, C Challis, JW Coenen, E De La Luna, et al. Physics of Plasmas (1994-present), 20(5):056111, 2013.

[6] RJ Goldston, DC McCune, HH Towner, SL Davis, RJ Hawryluk, and Schmidt GL. Journal of Computational Physics, 43(1):61-78, sep 1981.

[7] RJ Hawryluk et al. Physics of plasmas close to thermonuclear conditions, 1:19-46, 1980.

[8] T Dannert and F Jenko. Physics of Plasmas (1994-present), 12(7):072309, 2005.

[9] F Romanelli. Physics of Fluids B: Plasma Physics (1989-1993), 1(5):1018-1025, 1989.

[10] H Biglari, PH Diamond, and MN Rosenbluth. Physics of Fluids B: Plasma Physics (1989-1993), 1(1):109-118, 1989.

[11] BB Kadomtsev and OP Pogutse. Nuclear Fusion, 11(1):67, 1971.

[12] B Coppi and F Pegoraro. Nuclear Fusion, 17(5):969, 1977.

[13] W Horton Jr, D Choi, and WM Tang. Physics of Fluids (1958-1988), 24(6):1077-1085, 1981.

[14] PN Guzdar, L Chen, WM Tang, and PH Rutherford. Physics of Fluids (1958-1988), 26(3):673677, 1983.

[15] F Jenko, W Dorland, M Kotschenreuther, and BN Rogers. Physics of Plasmas (1994-present), 7(5):1904-1910, 2000.

[16] F Merz. Gyrokinetic simulation of multimode plasma turbulence. PhD thesis, University of Münster, 2009.

[17] RL Miller, MS Chu, JM Greene, YR Lin-Liu, and R. E. Waltz. Physics of Plasmas (1994-present), 5(4):973-978, 1998.

[18] LL Lao, H St. John, RD Stambaugh, AG Kellman, and W Pfeiffer. Nuclear Fusion, 25(11):1611, 1985.

[19] C Giroud, GP Maddison, S Jachmich, F Rimini, MNA Beurskens, I Balboa, S Brezinsek, R Coelho, JW Coenen, Lorenzo Frassinetti, et al. Nuclear Fusion, 53(11):113025, 2013.

[20] J Citrin, J Garcia, T Görler, F Jenko, P Mantica, D Told, C Bourdelle, DR Hatch, GMD Hogeweij, T Johnson, et al. Plasma Physics and Controlled Fusion, 57(1):014032, 2015.

[21] J Citrin, F Jenko, P Mantica, D Told, C Bourdelle, R Dumont, J Garcia, JW Haverkort, GMD Hogeweij, T Johnson, et al. Nuclear Fusion, 54(2):023008, 2014.

[22] P Mantica, C Angioni, C Challis, G Colyer, Lorenzo Frassinetti, N Hawkes, Thomas Johnson, M Tsalas, J Weiland, B Baiocchi, et al. Physical review letters, 107(13):135004, 2011. 